\title{
CREENCIAS DE AUTOEFICACIA Y ELECCIÓN FEMENINA DE ESTUDIOS CIENTÍFICO-TECNOLÓGICOS: UNA REVISIÓN TEÓRICA DE SU RELACIÓN
}

\author{
Self-efficacy beliefs and female choices of scientific- \\ technological studies: a theoretical review of their relationship \\ La croyance de l'auto-efficacité et des choix des femmes \\ d'études de la recherche scientifique et technologique: \\ un examen théorique de leur relation
}

M. ${ }^{a}$ del Carmen Rodríguez MÉndez, José Vicente Peña Calvo y Mercedes IndA CXARO

Universidad de Oviedo. Facultad de Formación del Profesorado y Educación. Departamento de Ciencias de la Educación. Despacho 318. C/ Aniceto Sela, s.n. 33005. Oviedo (España). Correo-e: carmenrm@uniovi.es; vipe@uniovi.es y indamaria@uniovi.es

Fecha de recepción: enero de 2012

Fecha de aceptación definitiva: abril de 2012

Biblid [(1130-3743) 24, 1-2012, 81-104]

RESUMEN

Las mujeres son mayoría en todos los niveles de enseñanza; sin embargo, todavía existen diferencias en la elección de determinadas opciones académicas. En el caso de la universidad están infrarrepresentadas en los estudios científicotecnológicos. Tomando en consideración esta situación, en el artículo realizamos un desarrollo teórico sobre las investigaciones que han analizado la influencia de las creencias de autoeficacia en la elección vocacional de las estudiantes. Estudios realizados en otros países han constatado que esta variable tiene un peso específico 
muy importante en dicha elección. De modo que aquellas estudiantes que se creen competentes en el dominio científico-tecnológico están más dispuestas a seleccionar carreras de esta rama, trabajan más duramente para completar las actividades con éxito y perseveran cuando se encuentran ante dificultades. Por ello, se indica que las creencias de autoeficacia son una variable relevante para explicar las elecciones académicas y el desarrollo de la carrera de las mujeres.

Palabras clave: género, creencias de autoeficacia, elección vocacional, estudios científico-tecnológicos.

\section{SUMMARY}

Women are the majority in all levels of education, but there are still differences in the choice of academic options. They are underrepresented in scientific and technological studies at the University. Considering this situation as an starting point, the paper presents a theoretical development over studies that have analyzed the influence of self-efficacy beliefs in career development of students. Studies have found that this variable has a very specific and important weight in this selection. Those students who think themselves competent in the domain of science and technology are more likely to select careers in this field, work harder to complete the activities successfully and persevere when faced with difficulties. Therefore, it indicates that self-efficacy beliefs are an important variable to explain the academic choices and career development of women.

Key words: gender, self-efficacy beliefs, career development, scientific and technological studies.

\section{SOMMAIRE}

Les femmes sont majoritaires dans tous les niveaux d'enseignement, cependant, il y a toujours des différences dans le choix de certaines options académiques. Dans le cas de l'université, les femmes sont sous-représentées dans les études scientifiques et technologiques. Considérant cette situation, cet article présente un développement théorique à partir des recherches qui ont analysé l'influence exercée par les croyances en l'auto-efficacité sur le choix de carrière des femmes. Des études menées dans d'autres pays ont constaté que cette variable a un poids spécifique très important lors de ce choix. C'est ainsi que les étudiantes qui ont le sentiment d'être compétentes dans le domaine scientifique et technologique sont plus disposées à choisir des études de ce secteur, vont travailler plus fort pour compléter les activités avec succès et vont persévérer malgré les difficultés. Par conséquent, on signale que les croyances en l'auto-efficacité constituent une variable importante pour expliquer les choix académiques et le développement de carrière des femmes.

Mots clés: genre, les croyances en l'auto-efficacité, choix de carrière, les études technologiques et scientifiques. 
1. DifERENCIAS DE GÉNERO EN LAS ELECCIONES ACADÉMICAS Y ORIENTACIÓN PARA LA CARRERA

La situación de las chicas en el sistema educativo ha experimentado un gran desarrollo en las últimas décadas, pues son mayoría en todos los niveles de enseñanza. Como ejemplo de esta situación señalamos que para el curso académico 2009/2010 el porcentaje de mujeres matriculadas en la universidad española ascendía al 54,3\% del total (MEC, 2012). Sin embargo, siguen persistiendo las diferencias de género en las elecciones académicas.

En las ramas del Bachillerato las chicas eligen, mayoritariamente, la opción de Humanidades y Ciencias Sociales; mientras que los chicos son mayoría en la rama Científico-Tecnológica (MEC, 2012; véase también Manassero y Vázquez, 2003; Rodríguez, Torío y Fernández, 2006; Vázquez y Manassero, 2008a). Asimismo, cuando se pregunta a chicos y chicas si les gustaría conseguir un trabajo en tecnología, ellos lo valoran positivamente y ellas de forma negativa; del mismo modo, las chicas rechazan más la vocación científico-tecnológica (Vázquez y Manassero, 2007, 2008a, 2008c, 2009). Respecto a la Formación Profesional, para el curso académico 2009/2010, se demostró que las mujeres se concentraron en las titulaciones de las áreas de Administración y Servicios Sociosanitarios, mientras que los varones lo hicieron en las especialidades con un mayor componente técnico y tecnológico (MEC, 2012).

Analizando los datos correspondientes a la universidad, para el curso académico 2007/2008 (MEC, 2012), observamos avances significativos, pero también algunos estancamientos en las posiciones. La mujeres son amplia mayoría en las carreras de Ciencias Experimentales, de ciclo largo $(56,5 \%)$ y corto $(73,6 \%)$; Ciencias de la Salud, de ciclo largo (70\%) y corto (79,2\%); Ciencias Sociales, de ciclo largo $(54,6 \%)$ y corto $(53,7 \%)$, y Humanidades (62\%). Por el contrario, su presencia es más exigua en las carreras Técnicas, de ciclo largo $(31,7 \%)$ y corto $(24,5 \%)$. Es más, respecto a los estudios de informática se constata que el número de mujeres decrece con el paso del tiempo (Fernández, Larraza, Maritxalar, Ruiz y Sarasola, 2008).

Estos resultados estadísticos también son corroborados en otros países. Investigaciones realizadas en Gran Bretaña muestran, consistentemente, que las mujeres siguen estando infrarrepresentadas en las carreras de ciencias físicas y matemáticas; así como en los estudios tecnológicos y de ingeniería (Colley y Comber, 2003; Durndell y Haag, 2002; Rodd y Bartholomew, 2006). En Australia, también se observa la menor presencia femenina en las carreras de físicas, matemáticas, ingeniería e informática (Anderson, Lankshear, Timms y Courtney, 2008; Leder y Forgasz, 2004); así como también se constata en Sudáfrica, los países escandinavos, Alemania o Israel (Ayalon, 2002, 2003; Bovée, Voogt y Meelissen, 2007; Brandell y Staberg, 2008; Nagy, Trautwein, Baumert, Köller y Garrett, 2006). La misma situación se mantiene en los EE. UU., donde hay pocas mujeres en carreras de ingeniería, informática, matemáticas o física; si bien en otras ciencias como la biología o la química no hay diferencias de género (Clark, 2005; Chipman, 2005; Frome, Alfeld, Eccles y Barber, 2006; Jacobs, 2005; Voyles y Williams, 2004; Zarret y Malanchuk, 2005). 
M. ${ }^{a}$ DEL C. RODRÍGUEZ MÉNDEZ, J. V. PEÑA CALVO Y M. INDA CXARO

CREENCIAS DE AUTOEFICACIA Y ELECCIÓN FEMENINA DE ESTUDIOS CIENTÍFICO-TECNOLÓGICOS

Asimismo, Ayalon (2003. Véanse también Bosch, Ferrer, Navarro y Seguí, 2008; Simpkins y Davis-Kean, 2005) constató que los chicos y chicas que habían cursado asignaturas de matemáticas en secundaria optaron por distintas carreras universitarias, de modo que ellos eligieron ingenierías y ciencias exactas, y las chicas medicina, derecho, administración de empresas y económicas.

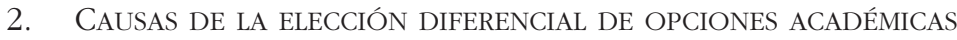

Del epígrafe anterior se extrae una conclusión rotunda que necesita ser analizada: las chicas eligen menos opciones académicas científico-tecnológicas. En consecuencia, en las páginas que siguen estudiaremos las causas que pueden influir en esta decisión vocacional de las mujeres.

\section{1. ¿Tienen las chicas menos éxito académico en las materias científico-tecnológicas?}

Se podría pensar que estas diferencias en la elección se deben a diferencias en el rendimiento. Así se dice que las chicas no eligen estas materias porque fracasan académicamente en los estudios científico-tecnológicos de la enseñanza secundaria. Sin embargo, se ha constatado que no hay diferencias de rendimiento en matemáticas (Bleeker y Jacobs, 2004; Brynes, 2005; Tiedemann, 2000; Watt, 2005); así como tampoco en ciencias naturales (Stewart, 1998) o en informática (Beyer, Rynes, Perrault, Hay y Haller, 2003; Coffin y MacIntrye, 1999; Fletcher-Flinn y Suddendorf, 1996). No obstante, otros estudios corroboraron que había diferencias de rendimiento en matemáticas, ciencias naturales o informática a favor de los chicos (Burkam, Lee y Smerdon, 1997; Joiner, Messer, Littleton y Light, 1996; Lapan, Shaughnessy y Boggs, 1996; Nagy, Trautwein, Baumert, Köller y Garrett, 2006). Otros estudios confirman que las chicas puntúan más que los chicos en matemáticas (Birenbaum y Nasser, 2006; Linver y Davis-Kean, 2005; Stewart, 1998), en informática (Papastergiou, 2008; Shashaani, 1997) o en ciencias naturales (Britner y Pajares, 2001, 2006; Stewart, 1998). También se ha indicado que hay diferencias en función del contenido matemático evaluado, en unos casos las chicas puntúan mejor y en otros lo hacen los chicos (Barbero, Holgado, Vila y Chacón, 2007; Wilson y Hart, 2001).

En el caso de las matemáticas, la disparidad de las conclusiones ha provocado una fuerte controversia. De este modo, la exhaustiva revisión bibliográfica realizada por Caplan y Caplan (2005) confirma, con rotundidad, que no existen diferencias y critica con dureza los fallos metodológicos de las investigaciones que sí las corroboran. Afirman que las diferencias en el rendimiento son muy pequeñas, inconsistentes y sólo aparecen cuando se trabaja con muestras muy seleccionadas y se realizan análisis estadísticos inusuales. Por tanto, al no haber diferencias, el rendimiento no es una de las causas de la elección vocacional que realizan las mujeres. 


\subsection{El uso del constructo "actitud" para explicar las diferencias en la elección académica}

La mayoría de los estudios demuestran que hay diferencias de género en las actitudes hacia las matemáticas, la informática o las ciencias experimentales/naturales, de modo que los varones puntúan más alto en las escalas que miden este constructo (Barbero, Holgado, Vila y Chacón, 2007; Durndell y Haag, 2002; Kadijevich, 2000; McIlroy, Bunting, Tierney y Gordon, 2001; Meelissen y Drent, 2008; Nosek, Banaji y Greenwald, 2002; Pierce, Stacey y Barkatsas, 2007; Rozell y Gardner, 2000). No obstante, algunos estudios no corroboran esta hipótesis y demuestran que chicos y chicas tienen actitudes positivas hacia estas materias (Bovée, Voogt y Meelissen, 2007; North y Noyes, 2002; Shaw y Marlow, 1999).

Pese a todo, los estudios que analizan la "actitud hacia..." han sido criticados porque el constructo se ha definido y operativizado de múltiples maneras y los instrumentos se han diseñado con poco fundamento empírico (Dryburgh, 2000; Fox y Soller, 2001; Manassero y Vázquez, 2002; Solvberg, 2002; Vázquez y Manassero, 1995, 1996, 2004; Vázquez, Manassero y Acevedo, 2005). Esto se percibe con claridad porque muchos artículos referencian la palabra "actitud" en el título, pero la lectura de su contenido revela que, bajo ese término, se ocultan conceptos distintos: intereses, motivación hacia el estudio, creencias de autoeficacia, ansiedad, utilidad percibida, etcétera.

Para solucionar los problemas conceptuales y metodológicos que se han descrito, las investigaciones de Vázquez y Manassero en España han sido muy cuidadosas en la definición del constructo y en las mediciones estadísticas que realizan. Sus estudios demuestran que los estudiantes de secundaria tienen una actitud general hacia las ciencias naturales/experimentales moderadamente positiva y revelan la tendencia a que las puntuaciones de los varones sean ligeramente superiores a las de las mujeres. Sin embargo, en la mayoría de los casos, estas diferencias tienen poca significatividad estadística por lo que la magnitud del efecto de la variable género es pequeña (Manassero y Vázquez, 2003; Vázquez y Manassero, 1996, 2004, 2007, 2008a, 2008b, 2009). Sin embargo, los estudios de estos autores también confirman que las actitudes favorables hacia estas disciplinas decrecen con la llegada de la adolescencia, pero este fenómeno es más acusado en las mujeres (Vázquez y Manassero, 2008a).

\section{3. "Las matemáticas no son cosa de chicas": la interiorización de estereotipos}

Por otra parte, diversos estudios han analizado la interiorización de prejuicios sexistas por parte de los chicos y chicas. Desde esta perspectiva, la mayoría de las investigaciones corroboran la ausencia de tales estereotipos en ellos y ellas (Durndell y Thomson, 1997; Forgasz, Leder y Kloosterman, 2004; Forgasz y Mittelberg, 2007; Rasmussen, 1997; Reinen y Plomp, 1997; Papastergiou, 2008). Sí hay mayor unanimidad en la conclusión de que ellas están más dispuestas a 
considerar que las matemáticas o la tecnología no son "masculinas" y que también pueden dedicarse a estas profesiones (Brandell y Staberg, 2008; Dryburgh, 2000; Keller, 2001; Johnson, Stone y Phillips, 2008; Meelissen y Drent, 2008; North y Noyes, 2002; Shashaani y Khalili, 2001).

Si tomamos en cuenta esta última conclusión, que es prácticamente unánime, se demuestra la paradoja Yo no puedo, pero nosotras sí podemos. Esta paradoja indica que las chicas niegan el estereotipo, pero les cuesta identificarse personalmente con estas materias. No admiten la discriminación, pero se resisten a entrar en estos dominios disciplinares. Están convencidas de que las mujeres, como colectivo, son tan capaces como los hombres, pero personalmente no emprenden la carrera científico-tecnológica. Llegados a este punto, la siguiente pregunta que debemos hacer es: ¿Por qué las mujeres no sienten interés o creen no tener la capacidad intelectual necesaria para triunfar académicamente en estas disciplinas?

\subsection{Las creencias de autoeficacia y su influencia en la elección vocacional}

Para responder a la pregunta con la que finalizamos el apartado anterior, muchas investigaciones han analizado la influencia de las creencias de autoeficacia en la elección vocacional. Desde esta postura, se sugiere que tales creencias están relacionadas con los procesos de elección de los estudios académicos. La autoeficacia es una variable precisada y analizada por Bandura, y que se define como las creencias que tiene una persona sobre su habilidad para realizar con éxito las tareas de un dominio específico. Así, se ha demostrado que las creencias de autoeficacia influyen en las elecciones académicas de las personas porque optamos por materias en las que nos sentimos competentes y rechazamos aquellas en las que no lo percibimos. También determinan el esfuerzo que invertimos cuando realizamos actividades, de forma que ponemos más esfuerzo en aquellas tareas en las que nos sentimos competentes.

Igualmente, las personas formamos nuestras creencias de autoeficacia interpretando la información que obtenemos de cuatro fuentes o recursos fundamentales. Estas fuentes o recursos son los siguientes (Bandura, 1977, 1982, 1998):

1. Experiencias previas: es el recurso con mayor influencia en la formación de las creencias de autoeficacia. Los estudiantes realizan tareas e interpretan los resultados de sus acciones, y usan esta información para desarrollar creencias sobre su competencia para realizar tareas similares. Normalmente, experiencias previas de éxito ayudan a incrementar la autoeficacia; al contrario, las que conducen al fracaso provocan una disminución de la autoeficacia.

2. Modelado y aprendizaje vicario: los estudiantes también forman sus creencias de autoeficacia observando a otras personas realizando la tarea y comparando su propia competencia con la de esas personas. Para que 
este recurso ejerza su influencia, el modelo observado debe poseer características personales similares al observador.

3. Persuasión y apoyo social: este recurso supone la exposición a los juicios, verbales y no verbales, de otras personas significativas para el sujeto. La persuasión social positiva puede ayudar a aumentar las creencias sobre la propia competencia y, al contrario, los refuerzos y estímulos negativos pueden desarrollar el sentido de la propia incapacidad.

4. Estado de arousal: el estado emocional que experimenta una persona cuando contempla o realiza una tarea influye en la formación de las creencias de autoeficacia.

Por tanto, las personas elaboramos nuestras creencias de autoeficacia a través de la interpretación de la información que recibimos de los cuatro recursos mencionados. La relevancia de cada uno de ellos en la formación de tales creencias dependerá de la materia o actividad y del proceso de integración que efectúe cada persona. En este contexto, se ha indicado que las creencias de autoeficacia tienen un peso específico muy importante para explicar el desarrollo de la carrera de las mujeres. En los epígrafes que siguen analizaremos este fenómeno con mayor detalle.

\subsubsection{Diferencias de género en las creencias de autoeficacia en distintas disciplinas académicas}

El primer estudio que analizó la influencia de las creencias de autoeficacia para explicar las diferencias de género en las elecciones académicas fue realizado por Betz y Hackett (1981). Estas autoras corroboraron que los hombres se sentían competentes para profesiones como ingeniero, matemático, delineante o economista; mientras que las mujeres se percibían competentes para ocupaciones como profesora, dentista, psicóloga o secretaria. También se concluyó que en las ocupaciones "femeninas" los varones tenían creencias de autoeficacia más altas que las que tenían las mujeres para las ocupaciones "masculinas". Más recientemente, en el estudio de Bandura, Barbaranelli, Caprara y Pastorelli (2001) se demostró que los chicos tenían un alto sentido de la autoeficacia para las carreras de ciencias naturales y experimentales, matemáticas y tecnología; mientras que las chicas puntuaban más alto para carreras del campo educativo, social y sanitario.

En lo que se refiere a la influencia del género en las creencias de autoeficacia matemática, los resultados de más de 30 años de estudio no son concluyentes, aunque mayoritariamente se inclinan en una dirección. El primer estudio realizado por Fennema y Sherman en los años 70 determina que las chicas tienen menos confianza que los chicos en su capacidad para aprender matemáticas y muchos estudios posteriores confirman esta primera conclusión (Bandura, Barbaranelli, Caprara y Pastorelli, 2001; Bleeker y Jacobs, 2004; Campbell y Hackett, 1986; Keller, 2001; Lapan, Shaughnessy y Boggs, 1996; Linver y Davis-Kean, 2005; Lussier, 1996; 
M. ${ }^{a}$ DEL C. RODRÍGUEZ MÉNDEZ, J. V. PEÑA CALVO Y M. INDA CXARO

CREENCIAS DE AUTOEFICACIA Y ELECCIÓN FEMENINA DE ESTUDIOS CIENTÍFICO-TECNOLÓGICOS

Mendick, 2005; Mittelberg y Lev-Ari, 1999; Pajares y Miller, 1994; Pierce, Stacey y Barkatsas, 2007; Sax, 1994; Seegers y Boekaerts, 1996; Tiedemann, 2000; Vermeer, Boekaerts y Seegers, 2000; Watt, 2005, 2006; Wigfield, Eccles, Yoon, Harold, Arbreton, Freedman-Doan y Blumenfeld, 1997).

En el campo de la informática, ingeniería y tecnología, el primer estudio realizado en la década de los 80 corroboró que los varones tenían mayores creencias de autoeficacia en estas disciplinas (Miura, 1987) y otras investigaciones posteriores también lo confirman (Barkatsas, Kasimatis y Gialamas, 2009; Beyer, Rynes, Perrault, Hay y Haller, 2003; Cassidy y Eachus, 2002; Durndell y Haag, 2002; Durndell, Haag y Laithwaite, 2000; Gargallo, Suárez y Belloch, 2003; Margolis, Fisher y Miller, 2000; Johnson, Stone, Phillips, 2008; Leslie, McClure y Oaxaca, 1998; Meelissen y Drent, 2008; Pierce, Stacey y Barkatsas, 2007; Papastergiou, 2008; Rodríguez y Angulo, 2006; Shashaani y Khalili, 2001; Schumacher y Morahan-Martin, 2001; Solvberg, 2002; Vekiri y Chronaki, 2008; Zarret y Malanchuk, 2005).

Como podemos observar los estudios se inclinan por corroborar las diferencias de género en las creencias de autoeficacia para las matemáticas y la tecnología. Por ello, debemos concluir que es una variable determinante en el proceso de elección vocacional de las mujeres.

2.4.2. Diferencias de género en los recursos que configuran las creencias de autoeficacia

En páginas anteriores indicamos que las creencias de autoeficacia se forman a partir de la información obtenida de cuatro recursos fundamentales: experiencias previas, persuasión social, aprendizaje vicario y estado emocional. A continuación, analizamos la influencia de cada una de estas variables en la configuración de las creencias de autoeficacia de varones y mujeres respecto a las disciplinas científicotecnológicas.

\subsubsection{Experiencias previas}

Numerosos estudios han analizado las diferencias de género en el acceso y uso de la tecnología, en especial de ordenadores y aparatos electrónicos. Las investigaciones concluyen que los varones tienen más experiencia en el uso de estos aparatos, los usan más horas a la semana, leen más revistas de tecnología y tienen más ordenadores personales (Anguita y Ordax, 2000; Cassidy y Eachus, 2002; Dickhäuser y Stiensmeier-Pelster, 2002; Durndell y Haag, 2002; Gargallo, 2008; Margolis, Fisher y Miller, 2000; Meelissen y Drent, 2008; North y Noyes, 2002; Papastergiou, 2008; Papastergiou y Solomonidou, 2005; Rodríguez y Angulo, 2006; Schumacher y Morahan-Martin, 2001; Solvberg, 2002; Vale y Leder, 2004; Vekiri y Chronaki, 2008; Volman y Van Eck, 2001). Igualmente, los varones eligen más asignaturas de informática y tecnología, tanto en la secundaria 
como en la universidad, y dedican más horas semanales a actividades tecnológicas (Dickhäuser y Stiensmeier-Pelster, 2003; Gargallo, 2008; Reinen y Plomp, 1997; Shashaani, 1997; Whitley, 1996; Papastergiou, 2008).

Por todo ello, se concluye que la mayor experiencia previa crea las oportunidades necesarias para manejar con éxito las herramientas tecnológicas, lo que, a su vez, influye en las creencias de autoeficacia de chicos y chicas. De este modo, cuando los efectos de la experiencia previa fueron controlados, algunos estudios demostraron que la variable género no predecía las creencias de autoeficacia o que su influencia en la predicción era menor (Busch, 1995; Cassidy y Eachus, 2002; Colley, Gale y Harris, 1994; Comber, Colley, Hargreaves y Dorn, 1997; Nelson y Cooper, 1997; Solvberg, 2002). También se ha constatado que las mujeres con acceso al ordenador en casa tienen mejor rendimiento en informática, mientras que esto no se cumple con los varones (Dugdale, Dekoven y Ju, 1998).

Respecto a las matemáticas, diversos estudios demuestran que el número de cursos de matemáticas realizados influye en las creencias de autoeficacia, constatándose que los varones completan más cursos en la escuela secundaria y en la universidad (Fox y Soller, 2001; Lamb, 1996; Lapan, Shaughnessy y Boggs, 1996; Wilson y Hart, 2001). Este hecho influye en la explicación de las diferencias de género en las creencias de autoeficacia matemática (Hackett, 1985; Lapan, Shaughnessy y Boggs, 1996; Lussier, 1996; Shashaani, 1995).

Asimismo, también se ha constatado que la atribución de las causas del éxito o fracaso en una actividad influye en las creencias de autoeficacia. Respecto a la atribución de éxito, se ha comprobado que los varones atribuyen su éxito matemático o en el uso de las tecnologías a su habilidad o talento innato; y las mujeres a factores tales como la ayuda docente, la suerte o el esfuerzo realizado (Campbell, 1990; Campbell y Hackett, 1986; Dickhäuser y Stiensmeier-Pelster, 2003; Forgasz y Leder, 1996; Hyde, Fennema, Ryan, Frost y Hopp; 1990; Nelson y Cooper, 1997; Seegers y Boekaerts, 1996; Watt, 2004, 2006). Del mismo modo, los chicos perciben, más que las chicas, que tendrán éxito en matemáticas y que la dificultad de las tareas encomendadas será menor (Watt, 2004, 2006).

En cuanto a la atribución de fracaso, se ha verificado que las chicas atribuyen sus fallos matemáticos o informáticos a su falta de capacidad (Dickhäuser y Stiensmeier-Pelster, 2003; Seegers y Boekaerts, 1996; Vermeer, Boekaerts y Seegers, 2000; Voyles y Williams, 2004); mientras que los chicos lo atribuyen a la mala suerte, la dificultad de la tarea o la falta de esfuerzo (Forgasz y Leder, 1996; Nelson y Cooper, 1997; Voyles y Williams, 2004). En línea similar, las chicas indican que tienen dificultades para comprender y usar los programas informáticos (Reinen y Plomp, 1997) y que el aprendizaje de esta materia es difícil (Shashaani, 1997).

Los estudios cualitativos confirman estos resultados al poner de manifiesto que los chicos están muy seguros de su habilidad o talento para la tecnología o las matemáticas, se sienten cómodos y seguros en estas materias y no atribuyen sus fallos a su falta de habilidad. Sin embargo, las chicas muestran claras reservas 
acerca de su competencia, atribuyen los problemas con las tareas a su incapacidad y sus éxitos a la buena suerte (Erwin y Maurutto, 1998; Volman, 1997; Zeldin, Britner y Pajares, 2008).

\subsubsection{Persuasión y apoyo social}

Como ya se ha indicado, otro recurso que influye en el desarrollo de las creencias de autoeficacia es la persuasión social. Puesto que la sociedad ha definido los patrones de conducta socialmente deseables para cada uno de los sexos, varones y mujeres reciben mensajes positivos y negativos cuando realizan determinadas elecciones académicas. Estos mensajes provienen del entorno social y son los progenitores y docentes quienes ejercen la mayor influencia a través de los mecanismos de persuasión social. Así, por ejemplo, el clásico estudio de Alemany, con estudiantes de ingeniería, indica que "mientras la mayoría de los alumnos logra una mayor cohesión familiar mediante su opción profesional, para algunas alumnas esa misma elección representa una relativa ruptura con el proyecto familiar" (Alemany, 1992, 40).

Se ha demostrado que los progenitores tienen distintas expectativas y aspiraciones respecto a las capacidades y rendimiento de los niños y niñas, lo que, a su vez, influye en las creencias de autoeficacia de unos y otras (Bandura, Barbaranelli, Caprara y Pastorelli, 2001; Fredricks y Eccles, 2002; Jacobs, Chhin y Bleeker, 2006; Linver y Davis-Kean, 2005; Wigfield, Eccles, Yoon, Harold, Arbreton, FreedmanDoan y Blumenfeld, 1997). Así, se ha verificado que cuando padres y madres mantienen la expectativa de que sus hijas deben elegir carreras "femeninas", éstas manifiestan expectativas similares que se hacen efectivas con el paso de los años (Jacobs, Chhin y Bleeker, 2006).

Con respecto a las matemáticas, se indica que las expectativas, creencias y actitudes de los progenitores ejercen una gran influencia en las actitudes y el rendimiento matemático de los niños y niñas (Zhongjuin, Bishop y Forgasz, 2006). En cuanto al género, se ha concluido que los padres y madres creen que los niños son mejores que las niñas en matemáticas, de modo que ellos tienen una actitud más positiva hacia las matemáticas porque cuentan con más apoyo parental (Tiedemann, 2000). Del mismo modo, Bleeker y Jacobs (2004) indican que las madres de los chicos tienen más altas expectativas del éxito matemático de sus hijos que las madres de las chicas y que si una madre tiene una baja percepción de la habilidad matemática de su hija, ésta tendrá bajas creencias de autoeficacia y estará menos dispuesta a planear una carrera en el ámbito científico-tecnológico. Igualmente se ha corroborado que padres y madres con fuertes estereotipos de género tienen más altas expectativas respecto a las habilidades y el rendimiento matemático de sus hijos que de sus hijas (Jacobs, 1991; Tiedemann, 2000); y que cuando las madres tienen menos estereotipos de género, unos y otras están más dispuestos a interesarse por las matemáticas, y cuando el padre tiene una visión estereotipada, el interés de las niñas disminuye y el de los niños aumenta (Jacobs, Davis-Kean, 
Bleeker, Eccles y Malanchuk, 2005). En conclusión, podemos decir que las expectativas parentales influyen en las oportunidades de aprendizaje que proporcionan a chicos y chicas, así como en las creencias que tienen sobre su capacidad y rendimiento matemático.

En lo que respecta a la tecnología, el patrón es similar pues chicos y chicas perciben que sus progenitores refuerzan y proporcionan más soporte a los varones que a las mujeres (Busch, 1995; Dickhäuser y Stiensmeier-Pelster, 2003; Meelissen y Drent, 2008; Reinen y Plomp, 1997; Shashaani, 1993, 1994, 1997; Shashaani y Khalili, 2001; Vekiri y Chronaki, 2008). Además, estas creencias parentales influyen en las decisiones adoptadas respecto a la carrera académica (Beyer, Rynes, Perrault, Hay y Haller, 2003; Shashaani, 1997; Shashaani y Khalili, 2001), y en sus intereses y creencias de autoeficacia. Los y las estudiantes que reciben apoyo y refuerzo parental usan más los aparatos tecnológicos, están más interesados en ellos, tienen creencias de autoeficacia más positivas y están más convencidos de su valor para resolver problemas de la vida cotidiana (Shashaani, 1994, 1997; Shashaani y Khalili, 2001; Vekiri y Chronaki, 2008).

De los estudios cualitativos se obtienen resultados complementarios que confirman estas ideas. Así, se indica que muchas mujeres que han realizado carreras universitarias en el dominio científico-tecnológico reconocen la relevancia del apoyo de personas allegadas para elegir y perseverar en su carrera académica (Clegg y Trayhurn, 1999; Erwin y Maurutto, 1998; Scott y Mallinckrodt, 2005; Zeldin y Pajares, 2000). Del mismo modo Lent et al. (2005) constatan que las mujeres que estudian ingeniería puntúan más alto que sus compañeros en la medida "apoyo social" y tienen similares creencias de autoeficacia. Ello demuestra la importancia que tiene para las mujeres el apoyo y refuerzo social en el desarrollo de las creencias de autoeficacia.

En lo que respecta a los docentes, también se obtienen resultados interesantes que demuestran su importancia como agentes implicados en el desarrollo de las creencias de autoeficacia de sus estudiantes. Así, se ha comprobado que perciben las matemáticas como un dominio masculino, tienen más bajas expectativas del rendimiento matemático/tecnológico de las chicas y proveen más persuasión verbal a los chicos para que aprendan matemáticas, lo que, a su vez, influye en sus creencias de autoeficacia (estudios citados en Fennema, Peterson, Carpenter y Lubinski, 1990. Véanse también Bleeker y Jacobs, 2004; Byrnes, 2005; Forgasz y Leder, 1996; Mittelberg y Lev-Ari, 1999; Tiedemann, 2000).

Igualmente se ha corroborado que los niños tienden a ser elegidos por sus docentes como los estudiantes más competentes en matemáticas. Además, atribuyen el alto rendimiento de las chicas a su esfuerzo, mientras que el de los chicos se atribuye, predominantemente, a su capacidad o talento innato. Igualmente, los docentes consideran que los chicos que son buenos en matemáticas son lógicos, persistentes y competitivos y que las chicas con éxito también lo son, pero en menor grado. Asimismo se considera que ellos exhiben un aprendizaje más autónomo, son más independientes y disfrutan más con las matemáticas (Fennema, Peterson, Carpenter y Lubinski, 1990). 
Todo ello se ve corroborado por las conclusiones de los estudios cualitativos pues demuestran que las chicas aumentan sus creencias de autoeficacia cuando perciben el apoyo de sus docentes y sienten que creen en su competencia científico-tecnológica (Clegg y Trayhurn, 1999; Dryburgh, 2000; Hanor, 1998; Jones y Jones, 1989; Margolis, Fisher y Miller, 2000; Roger y Duffield, 2000; Zeldin y Pajares, 2000). Asimismo, también hay estudios que demuestran que un ambiente escolar carente de apoyo y refuerzos positivos provoca una disminución de las creencias de autoeficacia e, incluso, el abandono de los estudios (Hanor, 1998; Herzig, 2004; Zeldin y Pajares, 2000; Wolffensperger, 1993).

\subsubsection{Modelado y aprendizaje vicario}

El tercer recurso que influye en el desarrollo de las creencias de autoeficacia es el aprendizaje vicario. Los adultos exhiben conductas que los niños y niñas pueden imitar y adoptar como parte de su repertorio comportamental. Desde este punto de vista, para los varones están disponibles muchos modelos que han tenido éxito en las matemáticas o la tecnología; además, en los centros escolares, la mayoría de los docentes de la rama científico-tecnológica son varones (Clark, 2005; Fox y Soller, 2001; Reinen y Plomp, 1997). Así, las mujeres científicas entrevistadas por Erwin y Maurutto (1998) lamentan no haber tenido profesoras que hubieran actuado como modelos de apoyo y estímulo a sus elecciones académicas. Del mismo modo, se observó que cuando las chicas tenían una profesora de informática, su actitud hacia esta materia era más positiva (Meelissen y Drent, 2008). Otras investigaciones concluyeron que estudiar matemáticas en una institución con muchas mujeres influía positivamente en las creencias de autoeficacia matemática (Sax, 1994) y que haber tenido modelos positivos aumentaba dichas creencias para persistir y tener altas aspiraciones académicas en los dominios científico-tecnológicos (Nauta, Epperson y Kahn, 1998).

En lo que se refiere a la influencia de modelos familiares, en el estudio cualitativo de Baker y Leary (1995) se constató que muy pocas chicas podían citar nombres de personas de su familia dedicadas a las ciencias naturales/experimentales y las que tenían algún pariente cercano estaban más dispuestas a considerar la posibilidad de ser científicas en este campo. De forma complementaria, el estudio de Erwin y Maurutto (1998), basado en entrevistas a mujeres que cursaban estudios de ciencias naturales/experimentales, demostró que el 36\% de ellas tenían, al menos, un progenitor con una carrera en este campo. En el estudio de López (1995), que comparó a estudiantes de ingeniería y pedagogía, se comprobó que el $32 \%$ de las chicas que realizaban ingeniería tenían tradición familiar en esta profesión, mientras que lo mismo sólo era cierto para el 12\% de los chicos.

Finalmente, destacamos las investigaciones de Pajares, Britner y Zeldin porque abordan el estudio de las creencias de autoeficacia a través del uso de entrevistas en profundidad con mujeres y varones que han cursado carreras de ciencias naturales/ 
experimentales. De sus estudios se ratifica que la persuasión social y el modelado han tenido una gran influencia en la formación de las creencias de estas mujeres, pues reconocen que algunas personas de su entorno han jugado un rol fundamental en su elección académica. El apoyo recibido y el modelado las han ayudado a ser más resistentes, a superar los obstáculos académicos y sociales y a contrarrestar los mensajes negativos que les envía el entorno social. Para ellas ha sido tan importante creer en sus potencialidades como que otras personas de su entorno también creyeran en ellas. Así, suelen tener varios familiares dedicados al trabajo científico-tecnológico y relatan experiencias de refuerzo y estímulo constante por parte de su familia y algunos docentes para persistir en su carrera (Zeldin y Pajares, 2000; Zeldin, Britner y Pajares, 2008).

Si bien los modelos estadísticos verifican que las experiencias previas son el recurso más influyente en la formación de las creencias de autoeficacia (Fouad, Smith y Zao, 2002; Lent, López, Brown y Gore, 1996); los estudios cualitativos de Pajares y colaboradores concluyen que, en el caso de estas mujeres, la persuasión y el modelado tienen un papel crucial (Zeldin y Pajares, 2000. Véase también Nauta, Epperson y Kahn, 1998). De este modo, los varones también reconocen la influencia positiva de estos recursos, pero no creen que hayan sido determinantes. En su caso, el apoyo recibido refuerza un sentido de la autoeficacia ya creado a partir de experiencias previas de éxito en actividades científico-tecnológicas. Posiblemente las mujeres estén más dispuestas a permitir que otras personas significativas jueguen un papel importante en la formación de sus creencias de autoeficacia.

\subsubsection{Estado de arousal}

El último recurso que influye en el desarrollo de las creencias de autoeficacia es el estado de arousal, pues se ha comprobado que si hay mucha ansiedad cuando se realiza una tarea se desarrollan unas pobres creencias de autoeficacia. En el tema que nos ocupa, se ha concluido que las mujeres manifiestan más ansiedad que los varones cuando desempeñan actividades científico-tecnológicas (Frost, Hyde y Fennema, 1994; Lent, López, Brown y Gore, 1996; Pajares y Miller, 1994) y cuando manejan las nuevas tecnologías (Chua, Chen y Wong, 1999; Coffin y McIntyre, 1999; Durndell y Haag, 2002; McIlroy, Bunting, Tierney y Gordon, 2001; Rozell y Gardner, 1999; Todman, 2000; Whitley, 1996).

En resumen, podemos concluir que las investigaciones demuestran que hay claras diferencias de género respecto a los cuatro recursos fundamentales que influyen en el desarrollo de las creencias de autoeficacia. A su vez, estas diferencias son determinantes para explicar las disparidades entre hombres y mujeres respecto a sus creencias de autoeficacia en el campo científico-tecnológico. De todo ello, concluimos resaltando el papel dominante que juegan tales creencias en el proceso de elección vocacional de las mujeres. 


\subsection{La influencia de otras variables: interés, utilidad y expectativas de resultados}

Si bien destacamos la influencia de las creencias de autoeficacia en la elección vocacional de las mujeres, también queremos subrayar el papel que juegan otras variables importantes. Así, en primer lugar, debemos considerar las diferencias de género en la percepción de interés por las materias científico-tecnológicas. Así, se ha comprobado que los varones disfrutan más y manifiestan más entusiasmo en las matemáticas o en el uso de la tecnología; y que las chicas tienden a considerar, más que los chicos, que la tecnología es aburrida y que no les gusta (Anderson, 2005; Anderson, Lankshear, Timms y Courtney, 2008; Kadijevich, 2000; Keller, 2001; Linver y Davis-Kean, 2005; Mcllroy, Bunting, Tierney y Gordon, 2001; Papastergiou, 2008; Red2Red Consultores, 2008; Rodríguez y Angulo, 2006; Vale y Leder, 2004; Watt, 2004, 2005, 2066).

En lo que respecta al valor de utilidad, algunos estudios demuestran que los chicos perciben la importancia para su futuro de materias como las nuevas tecnologías o las matemáticas (Fredricks y Eccles, 2002; Pelster, 2003; Nagy, Trautwein, Baumert, Köller y Garrett, 2006; Simpkins y Davis-Kean, 2005; Vale y Leder, 2004; Vekiri y Chronaki, 2008; Volman, Van Eck, Heemskerk y Kuiper, 2005). Sin embargo, otros estudios verifican que no hay diferencias de género (Bovée, Voogt y Meelissen, 2007; Forgasz, Leder y Kloosterman, 2004; Jacobs, Lanza, Osgood, Eccles y Wigfield, 2002; Kadijevich, 2000; Red2Red consultores, 2008; Shashaani y Khalili, 2001). Asimismo, Watt (2006) constata que las chicas que valoran la utilidad de las matemáticas están más dispuestas que otras chicas a aspirar a carreras relacionadas con esta disciplina, mientras que los chicos ya aspiran a ellas cuando tienen una valoración media de su utilidad. En línea similar, Farmer, Wardrop y Rotella (1999) realizan un estudio con estudiantes de carreras de ciencias naturales/ experimentales y demuestran que el valor de utilidad no fue un predictor importante de la participación masculina en estas carreras; sin embargo, en el caso de las chicas esta variable fue determinante.

Por último, señalamos una variable: las expectativas de resultado, que debemos diferenciar de las creencias de autoeficacia. Este constructo se refiere a la valoración que hace una persona sobre los resultados que obtendrá al realizar una elección académica. Dicha valoración se hace en base a aspectos tales como los beneficios y costes asociados a la profesión, el orgullo, la satisfacción o la devaluación derivadas del trabajo personal, la facilidad para entrar en la profesión, etcétera. Así, por ejemplo, una alumna puede percibir que realizar la carrera de matemáticas le reportará tales o cuales beneficios (expectativas de resultado); sin embargo, puede no sentirse capaz de obtener buenos resultados en esta carrera (creencias de autoeficacia pobres o negativas). Pues bien, se ha comprobado que los chicos tienen más expectativas de resultado respecto a la informática (Coffin y MacIntyre, 1999), y la ciencia y la matemática (Fouad y Smith, 1996). 


\section{A MODO DE CONCLUSIÓN}

Del desarrollo teórico que se ha seguido hasta este momento se puede concluir que todavía hoy en día las mujeres están infrarrepresentadas en las opciones académicas científico-tecnológicas. Los resultados corroboran que esta escasa representación no se explica por su bajo rendimiento académico o porque consideren que son dominios masculinos. Podemos considerar que hay otras variables en juego, destacando entre ellas las creencias de autoeficacia. A su vez, también determinan la elección otras variables como el interés y el valor de utilidad percibido, así como las expectativas de resultado. En cuanto a la relación entre las variables que acabamos de señalar, la investigación efectuada hasta el momento no ha sido capaz de determinarla con exactitud y los estudios continúan realizándose, primordialmente, en el ámbito anglosajón.

No obstante parece que se constata la tendencia a que las creencias de autoeficacia influyan en las otras variables, por lo que juegan un papel primordial en el proceso de elección vocacional. Así, diversos estudios corroboran su influencia en el interés por una materia académica y la utilidad percibida de esta (Betz y Hackett, 1997; Ferry, Fouad y Smith, 2000; Fredricks y Eccles, 2002; Jacobs, Lanza, Osgood, Eccles y Wigfield, 2002; Lapan, Shaughnessy y Boggs, 1996; Larose, Ratelle, Guay, Senécal y Harvey, 2006; Luzzo, Hasper, Albert, Bibby y Martinelli, 1999; Simpkins y Davis-Kean, 2005).

Asimismo, Lent y colaboradores han establecido un modelo general (teoría social cognitiva de la carrera) que determina la relación entre las variables que hemos descrito, añadiendo una nueva: las metas. En este sentido, su modelo confirma nuevamente el papel preponderante que ejercen las creencias de autoeficacia y su influencia en la predicción del resto de las variables (Lent, Brown, Sheu, Schmidt, Brenner, Gloster, Wilkins, Schmidt, Lyons y Treistman, 2005. Véanse también Lent, Brown, Schmidt, Brenner, Lyons y Treistman, 2003; Lent, López, López y Sheu, 2008; Lent, Sheu, Singley, Schmidt, Schmidt y Gloster, 2008).

Todos estos resultados permiten estar de acuerdo con la aseveración de Bandura, Barbaranelli, Caprara y Pastorelli (2001), para quienes las creencias de autoeficacia tienen una mayor peso predictivo en las preferencias ocupacionales que las expectativas de resultado, los intereses o las metas. Si bien, también establecen que estas variables tiene un peso específico importante y que es necesario realizar más estudios para precisar la relación entre las variables mencionadas.

\section{REFERENCIAS BIBLIOGRÁFICAS}

Alemany, C. (1992) Yo no be jugado nunca con Electro-L. Alumnas en enseñanza superior técnica. Madrid, Ministerio de Asuntos Sociales.

ANDERSON, N. (2005) Redressing the gender imbalance in ICT professions: toward-state-level strategic approaches. Australian Educational Computing, 20 (2), 3-10. 
ANDERSON, N.; LANKSHEAR, C.; Timms, C. y COURTNEY, L. (2008) «Because it's boring, irrelevant and I don't like computers": why high school girls avoid professionally-oriented ICT subjects. Computers \& Education, 50, 1304-1318.

ANGUITA, R. y ORDAX, E. (2000) Las alumnas ante los ordenadores: estrategias y formas de trabajo en el aula. Comunicar, 14, 218-224.

Ayalon, H. (2002) Mathematics and science course taking among Arab students in Israel: a case of unexpected gender equality. Educational Evaluation and Policy Analysis, 24 (1), 63-80.

- (2003) Women and men go to university: mathematical background and gender differences in choice of field in higher education. Sex Roles, 48 (5/6), 277-290.

BAKER, D. y LEARY, R. (1995) Letting girls speak out about science. Journal of Research in Science Teaching, 32 (1), 3-27.

BANDURA, A. (1977) Self-efficacy: toward a unifying theory of behavioural change. Psychological Review, 84 (2), 191-215.

- (1982) Self-efficacy mechanism in human agency. American Psychologist, 37 (2), 122-147.

- (1997) Self-efficacy. The exercise of control. New York, Freeman and Company.

Bandura, A.; Barbaranelli, C.; Caprara, G. y Pastorelli, C. (2001) Self-efficacy beliefs as shapers of children's aspirations and career trajectories. Child Development, 72 (1), 187-206.

Barbero, M. I.; Holgado, F. P.; Vila, E. y Chacón, S. (2007) Actitudes, hábitos de estudio y rendimiento en matemáticas: diferencias por género. Psicothema, 19 (3), 413-421.

Barkatsas, A.; Kasimatis, K. y Gialamas, V. (2009) Learning secondary mathematics with technology: exploring the complex interrelationship between student's attitudes, engagement, gender and achievement. Computers \& Education, 52, 562-570.

BeTZ, N. E. у НАскETT, G. (1981) The relationship of career-related self-efficacy expectations to perceived career options in college women and men. Journal of Counseling Psychology, 28 (5), 399-410.

- (1997) Applications of self-efficacy theory to the career assessment of women. Journal of Career Assessment, 5 (4), 383-402.

Birenbaum, M. y NASSER, F. (2006) Ethnic and gender differences in mathematics achievement and in dispositions towards the study of mathematics. Learning and Instruction, $16,26-40$.

Bleeker, M. M. y Jacobs, J. (2004) Achievement in math and science: do mothers' beliefs matter 12 years later? Journal of Educational Psychology, 96 (1), 97-109.

Bosch, E.; Ferrer, V.; NAVArro, C. y Seguí, I. (2008) Toma de decisiones del alumnado de bachillerato en relación a los estudios científico-técnicos. Análisis de las diferencias según el género, en Miqueo, C.; Barral, M. J. y Magallón, C. (eds.) Estudios iberoamericanos de género en ciencia, tecnología y salud. Zaragoza, Prensas Universitarias de Zaragoza, 555-560.

Bovée, C.; Voogt, J. y Meelissen, M. (2007) Computer attitudes of primary and secondary in South Africa. Computers in Human Behavior, 23, 1762-1776.

BRANDELL, G. y STABERG, E.-M. (2008) Mathematics: a female, male or gender-neutral domain? A study of attitudes among students at secondary level. Gender and Education, 20 (5), 495-509.

Britner, G. y Pajares, F. (2001) Self-efficacy beliefs, motivation, race, and gender in middle school science. Journal of Women and Minorities in Science and Engineering, 7, 269-283.

- (2006) Sources of science self-efficacy beliefs of middle school students. Journal of Research in Science Teaching, 43 (5), 485-499. 
BRYNES, J. P. (2005) Gender differences in math. Cognitive processes in an expanded framework, en Gallagher, A. M. y Kaufman, J. Gender differences in mathematics. An integrative psychological approach. New York, Cambridge University Press, 73-98.

Busch, T. (1995) Gender differences in self-efficacy and attitudes toward computers. Journal of Educational Computing Research, 12 (2), 147-158.

CAMPBELl, N. (1990) High school student's computer attitudes and attributions: gender and ethnic group differences. Journal of Adolescent Research, 5 (4), 485-499.

CAMPBELl, N. K. y HACKETT, G. (1986) The effects of mathematics task performance on math self-efficacy and task interest. Journal of Vocational Behavior, 28, 149-162.

CaPlan, J. y CaPlan, P. (2005) The perseverative search for sex differences in mathematics ability, en Gallagher, A. M. y Kaufman, J. Gender differences in mathematics. An integrative psychological approach. New York, Cambridge University Press, 25-47.

Cassidy, S. y Eachus, P. (2002) Developing the computer user self-efficacy (cuse) scale: investigating the relationship between computer self-efficacy, gender and experience with computers. Journal of Educational Computing Research, 26 (2), 133-153.

Chipman, S. F. (2005) Research on the women and mathematics issue. A personal case history, en Gallagher, A. M. y Kaufman, J. Gender differences in mathematics. An integrative psychological approach. New York, Cambridge University Press, 1-24.

Chua, S. L.; Chen, D.-T. y Wong, A. F. L. (1999) Computer anxiety and its correlates: a metaanalysis. Computers in Human Behavior, 15, 609-623.

Clark, J. (2005) Women and science careers: leaky pipeline or gender filter? Gender and Education, 17 (4), 369-386.

Clegg, S. y Trayhurn, D. (1999) Gender and computing: not the same old problem. British Educational Research Journal, 26 (1), 75-89.

Coffin, R. J. y MaCINTYRE, P. D. (1999) Motivational influences on computer-related affective states. Computers in Human Behavior, 15, 549-569.

COlley, A. y COMBER, C. (2003) School subject preferences: age and gender differences revisited. Educational Studies, 29 (1), 59-67.

Colley, A. M.; Gale, M. T. y Harris, T. A. (1994) Effects of gender role identity and experience on computer attitude components. Journal of Educational Computing Research, 10 (2), 129-137.

Comber, C.; Colley, A.; Hargreaves, D. J. y Dorn, L. (1997) The effects of age, gender and computer experience upon computer attitudes. Educational Research, 39 (2), 123-133.

Dickhäuser, O. y Stiensmeier-Pelster, J. (2002) Gender differences in computer work: evidence for the model of achievement-related choices. Contemporary Educational Psychology, 27, 486-496.

- (2003) Gender differences in the choice of computer courses: applying and expectancyvalue model. Social Psychology of Education, 6, 173-189.

DRYBURGH, H. (2000) Underrepresentation of girls and women in computer science: classification of 1990s research. Journal of Educational Computing Research, 23 (2), 181-202.

Dugdale, S.; Dekoven, E. y Ju, M. (1998) Computer course enrollment, home computer access, and gender: relationships to high school student's success with computer spreadsheet use for problem solving in pre-algebra. Journal of Educational Computing Research, 18 (1), 49-62.

Durndell, A. y HAAG, Z. (2002) Computer self efficacy, computer anxiety, attitudes towards the Internet and reported experience with the Internet, by gender, in an east European sample. Computers in Human Behavior, 18, 521-535. 
Durndell, A.; HaAg, Z. y Laithwaite, H. (2000) Computer self efficacy and gender: a cross cultural study of Scotland and Romania. Personality and Individual Differences, 28, 1037-1044.

Durndell, A. y Thomson, K. (1997) Gender and computing: a decade of change? Computers E Education, 28 (1), 1-9.

ERWIN, L. y MAUTURRO, P. (1998) Beyond access: considering gender deficits in science education. Gender and Education, 10 (1), 51-69.

FARMER, H. S.; WARDROP, J. L. y ROTELla, S. C. (1999) Antecedent factors differentiating women and men in science/nonscience careers. Psychology of Women Quarterly, 23, 763-780.

Fennema, E.; Peterson, P. L.; Carpenter, T. P. y Lubinski, C. A. (1990) Teacher's attributions and beliefs about girls, boys, and mathematics. Educational Studies in Mathematics, 21, 55-69.

Fernández, V.; Larraza, E.; Maritxalar, M.; Ruiz, T. y SARasola, K. (2008) Ingeniería en informática y género: un estudio cuantitativo, en Miqueo, C.; BARral, M. J. y Magallón, C. (eds.) Estudios iberoamericanos de género en ciencia, tecnología y salud. Zaragoza, Prensas Universitarias de Zaragoza, 629-638.

FERRY, T. R.; FouAd, N. A. y SMITH, P. L. (2000) The role of family context in a social cognitive model for career related choice behavior: a math and science perspective. Journal of Vocational Behavior, 57, 348-364.

FletCher-Flinn, C. M. y SudDENDORF, T. (1996) Computer attitudes, gender and exploratory behavior: a developmental study. Journal of Educational Computing Research, 15 (4), 389-392.

Forgasz, H. y Leder, G. (1996) Mathematics classrooms, gender and affect. Mathematics Education Research Journal, 8 (1), 153-173.

Forgasz, H.; Leder, G. y KloOsterman, P. (2004) New perspectives on the gender stereotyping of mathematics. Mathematical Thinking and Learning, 6 (4), 389-420.

Forgasz, H. y MitTELBERG, D. (2007) Gendered beliefs about mathematics among Australian and Israeli grade 9 students. Consultado el 11 de julio de 2011. http://www.aare.edu. au/07pap/for07212.pdf.

Fouad, N. A. y Smith, P. L. (1996) A test of a social cognitive model for middle school students: math and science. Journal of Counseling Psychology, 43 (3), 338-346.

Fouad, N. A.; Smith, P. L. y ZaO, K. E. (2002) Across academic domains: extensions of the social-cognitive career model. Journal of Counseling Psychology, 49 (2), 164-171.

Fox, L. H. y Soller, J. F. (2001) Psychological dimensions of gender differences in mathematics, en JACOBS, J.; BeCKer, J. y GiLMer, G. (eds.) Changing the faces of mathematics. Virginia: National Council of Teachers of Mathematics, 9-24.

FREDRICKS, J. A. y ECCLES, J. S. (2002) Children's competence and value beliefs from childhood through adolescence: growth trajectories in two male-sex-typed domains. Developmental Psychology, 38 (4), 519-533.

Frome, P.; Alfeld, C.; Eccles, J. y Barber, B. (2006) Why don't they want a male-dominated job? An investigation of young women who changed their occupational aspirations. Educational Research and Evaluation, 12 (4), 359-372.

Frost, L. A.; Hyde, J. S. y FenNema, E. (1994) Gender, mathematics performance, and mathematics-related attitudes and affect: a meta-analytic synthesis. International Journal of Educational Research, 21, 373-385. 
Gargallo, B. (2008) Género y nuevas tecnologías, en AzNAR, P. y CÁnOvas, P. (eds.) Educación, género y politicas de igualdad. Valencia, Universitat de Valencia, 255-282.

Gargallo, B.; Suárez, J. y Belloch, C. (2003) La división digital en el proceso de integración de las NTC en la educación. Diferencias de género entre alumnos de ESO de la comunidad valenciana. Teoría de la Educación: Educación y Cultura en la Sociedad de la Información, 4. Consultado el 14 de julio de 2011. http://campus.usal. es/ teoriaeducacion/rev_numero_04/n4_art_gargallo-suarez-belloch.htm.

HAскетT, G. (1985) Role of mathematics self-efficacy in the choice of math-related majors of college women and men: a path analysis. Journal of Counseling Psychology, 32 (1), 47-56.

HANOR, J. H. (1998) Concepts and strategies learned from girl's interactions with computers. Theory into Practice, 37 (1), 64-71.

Herzig, A. H. (2004) "Slaughtering this beautiful math": graduate women choosing and leaving mathematics. Gender and Education, 16 (3), 379-395.

Hyde, J. S.; Fennema, E.; Ryan, M.; Frost, L. A. y Hopp, C. (1990) Gender comparisons of mathematics attitudes and affect. Psychology of Women Quarterly, 14, 299-324.

JACOBS, J. E. (1991) Influence of gender stereotypes on parent and child mathematics attitudes. Journal of Educational Psychology, 83 (4), 518-527.

- (2005) Twenty-five years of research on gender and ethnic differences in math and science career choices: what have we learned? New Directions for Child and Adolescent Development, 110, 85-94.

JaCOBS, J. E.; ChHin, C. S. y BleEKer, M. M. (2006) Enduring links: parent's expectations and their young adult children's gender-typed occupational choices. Educational Research and Evaluation, 12 (4), 395-407.

Jacobs, J. E.; Davis-Kean, P.; Bleeker, M.; Eccles, J. S. y Malanchuk, O. (2005) «I can, but I don't want to". The impact of parents, interests and activities on gender differences in math, en Gallagher, A. M. y Kaufman, J. C. Gender differences in mathematics. An integrative psychological approach. New York, Cambridge University Press, 246-263.

Jacobs, J. E.; LANZA, S.; OsGoOd, D. W.; ECCles, J. y Wigfield, A. (2002) Changes in children's self-competence and values: gender and domain differences across grades one through twelve. Child Development, 73 (2), 509-527.

Johnson, R. D.; Stone, D. L. y Philipps, T. N. (2008) Relations among ethnicity, gender, beliefs, attitudes, and intention to pursue a career in information technology. Journal of Applied Social Psychology, 38 (4), 999-1022.

Joiner, R.; Messer, D.; LitTleton, K. y Light, P. (1996) Gender, computer experience and computer-based problem solving. Computers \& Education, 26 (1-3), 179-187.

JONES, L. G. y JONES, L. P. (1989) Context, confidence and the able girl. Educational Research, 31 (3), 189-194.

KADIJEVICH, D. (2000) Gender differences in computer attitude among ninth-grade students. Journal of Educational Computing Research, 22 (2), 145-154.

KELLER, C. (2001) Effect of teachers' stereotyping on student's stereotyping of mathematics as a male domain. The Journal of Social Psychology, 14 (2), 165-173.

LAMB, S. (1996) Gender differences in mathematics participation in Australian schools: some relationships with social class and school policy. British Educational Research Journal, 22 (2), 223-240. 
Lapan, R. T.; Shaughnessy, P. y Boggs, K. (1996) Efficacy expectations and vocational interest as mediators between sex and choice of math/science college majors: a longitudinal study. Journal of Vocational Behavior, 49, 277-291.

larose, S.; Ratelle, C. F.; Guay, F.; SenéCal, C. y Harvey, M. (2006) Trajectories of science selfefficacy beliefs during the college transition and academic and vocational adjustment in science and technology programs. Educational Research and Evaluation, 12 (4), 373-393.

Lent, R. W.; BRown, S. D.; SCHMidT, J.; Brenner, B.; Lyons, H. y Treistman, D. (2003) Relation of contextual supports and barriers to choice behavior in engineering majors: test of alternative social cognitive models. Journal of Counseling Psychology, 50 (4), 458-465.

Lent, R.W.; Brown, S. D.; Sheu, H.; Schmidt, J.; Brenner, B.; Gloster, C.; Wilkins, G.; SCHMidT, L.; LyONS, H. y Treistman, D. (2005) Social cognitive predictors of academic interest and goals in engineering: utility for women and students at historically black universities. Journal of Counseling Psychology, 52 (1), 84-92.

LENT, R. W.; LÓPEZ, F. G.; BROWN, S. D. y Gore, P. A. (1996) Latent structure of the sources of mathematics self-efficacy. Journal of Vocational Behavior, 49, 292-308.

LenT, R. W.; LóPez, A. M.; López, F. G. y SHeu, H. (2008) Social cognitive career theory and the prediction of interests and choice goals in the computing disciplines. Journal of Vocational Behavior, 73, 52-62.

Lent, R. W.; Sheu, H.; Singley, D.; Schmidt, J.; Schmidt, L. y Gloster, C. (2008) Longitudinal relations of self-efficacy to outcome expectations, interest, and major choice goals in engineering students. Journal of Vocational Behavior, 73, 328-335.

Leslie, L. L.; MCCluRe, G. T. y OAXACA, R. L. (1998) Women and minorities in science and engineering. A life sequence analysis. The Journal of Higher Education, 69 (3), 239-276.

Linver, M. R. y DAVIS-KeAn, P. E. (2005) The slippery slope: what predicts math grades in middle and high school? New Directions for Child and Adolescent Development, 110, 49-64.

LóPez, M. (1995) La elección de una carrera típicamente femenina o masculina. Desde una perspectiva psicosocial: la influencia del género. Madrid, Ministerio de Educación y Ciencia.

Lussier, G. (1996) Sex and mathematical background as predictors of anxiety and selfefficacy in mathematics. Psychological Reports, 79, 827-833.

LuZzo, D. A.; HASPER, P.; Albert, K.; BibBy, M. y Martinelli, E. (1999) Effects of self-efficacyenhancing interventions on the math/science self-efficacy and career interest, and actions of career undecided college students. Journal of Counseling Psychology, 46 (2), 233-243.

Macilroy, D.; Bunting, K.; Tierney, K. y Gordon, M. (2001) The relation of gender and background experience to self-reported computing anxieties and cognitions. Computers in Human Behavior, 17, 21-33.

MANASSERO, M. A. y VÁZQUEZ, A. (2002) Estructura de las actitudes relacionadas con la ciencia y diferencias de género. Bordón, 54 (4), 587-604.

- (2003) Los estudios de género y la enseñanza de las ciencias. Revista de Educación, $330,251-280$.

Margolis, J.; Fisher, A. y Miller, F. (2000) The anatomy of interest: women in undergraduate computer science. Women's Studies Quarterly, 1-35.

MEC (2012) Estadísticas de la enseñanza universitaria y no universitaria. Consultado el 7 de noviembre de 2012. http://www.educacion.es/horizontales/documentacion/estadisticas.html. 
MeElissen, M. y Drent, M. (2008) Gender differences in computer attitudes: does the school matter? Computers in Human Behavior, 24, 969-985.

Mendick, H. (2005) A beautiful myth? The gendering of being/doing "good at math's". Gender and Education, 17 (2), 203-219.

MitTelBerg, D. y LeV-ARI, L. (1999) Confidence in mathematics and its consequences: gender differences among Israeli Jewish and Arab youth. Gender and Education, 11 (1), 75-92.

MiURA, I. T. (1987) The relationship of computer self-efficacy expectations to computer interest and course enrollment in college. Sex Roles, 16 (5/6), 303-311.

Nagy, G.; Trautwein, U.; Baumert, J.; Köller, O y Garrett, J. (2006) Gender and course selection in upper secondary education: effects of academic self-concept and intrinsic values. Educational Research and Evaluation, 12 (4), 323-345.

NAUTA, M. M.; EPPERSON, D. L. y KAHN, J. H. (1998) A multiple-groups analysis of predictors of higher level career aspirations among women in mathematics, science and engineering majors. Journal of Counseling Psychology, 45 (4), 483-496.

Nelson, L. J. y COOPER, S. J. (1997) Gender differences in children's reactions to success and failure with computers. Computers in Human Behavior, 13 (2), 247-67.

NORTH, A. S. y NOYES, J. M. (2002) Gender differences on children's computer attitudes and cognitions. Computers in Human Behavior, 18, 135-150.

Nosek, B. A.; BanajI, M. y Greenwald, A. G. (2002) Math=male, me=female, therefore math $\neq$ me. Journal of Personality and Social Psychology, 83 (1), 44-59.

PAJARES, F. y Miller, D. (1994) Role of self-efficacy and self-concept beliefs in mathematical problem solving: a path analysis. Journal of Counseling Psychology, 86 (2), 193-203.

PAPASTERgIOU, M. (2008) Are computer science and information technology still masculine fields? High school student's perceptions and career choices. Computers $\&$ Education, 51, 594-608.

PAPASTERgIOU, M. y SOlOMONIDOU, C. (2005) Gender issues in internet access and favorite internet activities among Greek high school pupils inside and outside school. Computers \& Education, 44, 377-393.

Pierce, R.; Stacey, K. y Barkatsas, A. (2007) A scale for monitoring student's attitudes to learning mathematics with technology. Computers E Education, 48, 285-300.

Rasmussen, B. (1997) Girls and computer science: It's not me. I'm not interested in sitting behind a machine all day, en Grundy, A. F.; KöHler, D.; Oechtering, V. y Petersen, O. (eds.) Women, work and computerization. Spinning a web from past to future. Proceedings of the $6^{\text {th }}$ International IFIP-Conference. Berlín, Springer, 379-386.

RED2RED CONSULTORES (2008) Mujeres y nuevas tecnologias de la información y la comunicación. Madrid, Instituto de la Mujer.

REINEN, I. J. y PLOMP, T. (1997) Information technology and gender equality: a contradiction in terminis. Computers \& Education, 28 (2), 65-78.

RODD, M. y BARTHOLOMEW, H. (2006) Invisible and special: Young women's experiences as undergraduate mathematics students. Gender and Education, 18 (1), 35-50.

Rodríguez, C. y Angulo, F. (2006) Problemas y limitaciones del acceso de las jóvenes a las tecnologías de la información y la comunicación, en Rodríguez, C. (comp.) Género y currículo. Aportaciones del género al estudio y práctica del currículo. Madrid: Akal, 131-152.

Roger, A. y Duffield, J. (2000) Factors underlying persistent gendered option choices in school science and technology in Scotland. Gender and Education, 12 (3), 367-383. 
ROzell, E. J. y GARDNER, W. L. (1999) Computer-related success and failure: a longitudinal study of the factors influencing computer-related performance. Computers in Human Behavior, 15, 1-10.

- (2000) Cognitive, motivation and affective processes associated with computer-related performance: a path analysis. Computers in Human Behavior, 16, 199-222.

Schumacher, P. y Morahan-Martin, J. (2001) Gender, Internet and computer attitudes and experiences. Computers in Human Behavior, 17, 95-110.

SCOTT, A. B. y MALLinckrodT, B. (2005) Parental emotional support, science self-efficacy, and choice of science major in undergraduate women. The Career Development Quarterly, 53, 263-273.

SEegers, G. y BOEKAERTS, M. (1996) Gender-related differences in self-referenced cognitions in relation to mathematics. Journal for Research in Mathematics Education, 27 (2), 215-240.

SHASHAAnI, L. (1993) Gender-based differences in attitudes toward computers. Computers $\mathcal{E}$ Education, 20 (2), 169-181.

- (1994) Socioeconomic status, parent's sex role stereotypes and the gender gap in computing. Journal of Research on Computing in Education, 26 (4), 433-451.

- (1995) Gender differences in mathematics experience and attitude and their relation to computer attitude. Educational Technology, 35 (3), 32-37.

- (1997) Gender differences in computer attitudes and use among college students. Journal of Educational Computing Research, 16 (1), 37-51.

SHASHAANI, L. y KHALILI, A. (2001) Gender and computers: similarities and differences in Iranian college students' attitudes toward computers. Computers \& Education, 37, 363-375.

SHAW, G. y MARLOW, N. (1999) The role of student learning styles, gender, attitudes and perceptions on information and communication technology assisted learning. Computers EEducation, 33, 223-234.

SimpKinS, S. D. y DAVIS-KeAN, P. E. (2005) The intersection between self-concepts and values: links between beliefs and choices in high school. New Directions for Child and Adolescent, 110, 31-47.

SolvberG, A. M. (2002) Gender differences in computer-related control beliefs and home computer use. Scandinavian Journal of Educational Research, 46 (4), 409-426.

STEWART, M. (1998) Gender issues in physics education. Educational Research, 40 (3), 283-293.

Tiedemann, J. (2000) Parent's gender stereotypes and teacher's beliefs as predictors of children's concept of their mathematical ability in elementary school. Journal of Educational Psychology, 92 (1), 144-151.

Todman, J. (2000) Gender differences in computer anxiety among university entrants since 1992. Computers \& Education, 34, 27-35.

Vale, C. M. y Leder, G. C. (2004) Student views of computer-based mathematics in the middle years: does gender make a difference. Educational Studies in Mathematics, 56, 287-312.

VÁzQuez, A. y Manassero, M. A. (1995) Actitudes relacionadas con la ciencia: una revisión conceptual. Enseñanza de las Ciencias, 13 (3), 337-346.

- (1996) Factores determinantes de las actitudes relacionadas con la ciencia. Revista Española de Pedagogía, 203, 43-77.

- (2004) Imagen de la ciencia y la tecnología al final de la educación obligatoria. Cultura y Educación, 16 (4), 385-398. 
- (2007) En defensa de las actitudes y emociones en la educación científica (II): evidencias empíricas derivadas de la investigación. Revista Eureka sobre Enseñanza y Divulgación de las Ciencias, 4 (3), 417-441.

- (2008a) La elección de asignaturas de ciencia: análisis de los factores determinantes. Revista Española de Pedagogía, 241, 541-558.

- (2008b) El declive de las actitudes hacia la ciencia de los estudiantes: un indicador inquietante para la educación científica. Revista Eureka sobre Enseñanza y Divulgación de las Ciencias, 5 (3), 274-292.

- (2008c) La vocación científica y tecnológica de las chicas en secundaria y la educación diferenciada. Bordón, 60 (3), 149-163.

- (2009) La relevancia de la educación científica: actitudes y valores de los estudiantes relacionados con la ciencia y la tecnología. Enseñanza de las Ciencias, 27 (1), 33-48.

VÁzquez, A.; Manassero, M. A. y Acevedo, J. A. (2005) Análisis cuantitativo de ítems complejos de opción múltiple en ciencia, tecnología y sociedad: escalamiento de ítems. Revista Electrónica de Investigación Educativa, 781.

Vermeer, H.; Boekaerts, M. y Seegers, G. (2000) Motivational and gender differences: sixth-grade students' mathematical problem-solving behavior. Journal of Educational Psychology, 92 (2), 308-315.

Volman, M. (1997) Gender-related effects of computer and information literacy education. Journal of Curriculum Studies, 29 (3), 315-328.

Volman, M. y VAn ECK, E. (2001) Gender equity and information technology in education: the second decade. Review of Educational Research, 71, 613-634.

Volman, M.; VAn EcK, E.; HeEMSKerk, I. y KuIPer, E. (2005) New technologies, new differences. Gender and ethnic differences in pupil's use of ICT in primary and secondary education. Computers \& Education, 45, 35-55.

VOYLES, M. y Williams, A. (2004) Gender differences in attributions and behavior in a technology classroom. Journal of Computers in Mathematics and Science Teaching, 23 (3), 233-256.

WaTT, H. M. (2004) Development of adolescents' self-perceptions, values, and task perceptions according to gender and domain in 7th-through 11 th-grade Australian students. Child Development, 75 (5), 1556-1574.

- (2005) Explaining gendered math enrollments for NSW Australian secondary schools students. New Directions for Child and Adolescent, 110, 15-29.

- (2006) The role of motivation in gendered educational and occupational trajectories related to maths. Educational Research and Evaluation, 12 (4), 305-322.

WhitLey, B. E. (1996) Gender differences in computer-related attitudes: it depends on what you ask. Computers in Human Behavior, 12 (2), 275-289.

Wigfield, A.; Eccles, J.; Yoon, K.; Harold, R.; Arbreton, A.; Freedman-Doan, C. y BluMENFELD, P. (1997) Change in children's competence beliefs and subjective task values across the elementary school years: a 3-Year study. Journal of Educational Psychology, 89 (3), 451-469.

WILSON, P. y HART, L. (2001) Teachers as researchers. Understanding gender issues in mathematics education, en Jacobs, J.; Becker, J. y Gilmer, G. (eds.) Changing the faces of mathematics. Virginia, National Council of Teachers of Mathematics, 43-57.

WolfFensperger, J. (1993) "Science is truly a male world". The interconnectedness of knowledge, gender and power within university education. Gender and Education, 5 (1), 37-54 
ZARRET, N. R. y MALANChUK, O. (2005) Who's computing? Gender and race differences in young adult's decisions to pursue an information technology career. New Directions for Child and Adolescent Development, 110, 65-84.

Zeldin, A. L.; Britner, S. L. y Pajares, F. (2008) A comparative study of the self-efficacy beliefs of successful men and women in mathematics, science and technology careers. Journal of Research in Science Teaching, 45 (9), 1036-1058.

Zeldin, A. L. y PAJARES, F. (2000) Against the odds: self-efficacy beliefs of women in mathematical, scientific and technological careers. American Educational Research Journal, 37 (1), 215-246. 